\title{
Osteoarthritis and a high-fat diet: the full ' $O A$ syndrome' in a small animal model
}

\author{
Peter M van der Kraan* \\ See related research by Griffin et al., http://arthritis-research.com/content/12/4/R130
}

\begin{abstract}
Obesity is one of the main risk factors for osteoarthritis $(\mathrm{OA})$ and due to the global rise in obesity this will increasingly contribute to OA development. The article of Griffin and co-workers in this issue of Arthritis Research and Therapy shows that a high-fat diet leads to obesity and OA in the studied animals and that this is related to alterations in locomotor function. Furthermore, a high-fat diet leads to pain sensitization and depression/anxiety-like behavior unrelated to structural OA changes in the knee. Their findings demonstrate that the majority of features of the human 'OA syndrome' can be reproduced in a small animal model.
\end{abstract}

Osteoarthritis (OA) is the most common joint disease and affects a large proportion of the population, mainly the elderly. Besides aging, obesity is one of the most important risk factors for OA. Due to the global 'obesity epidemic', it is becoming more and more important to unravel the role of obesity in both structural and symptomatic OA. The article of Griffin and colleagues in Arthritis Research and Therapy is one of first studies to integrate in a high-fat-induced small animal model of OA the subsequent obesity and biochemical, neurobehavioral, musculoskeletal, inflammatory and structural knee joint changes [1]. Many aspects of the spectrum of structural and symptomatic findings in human OA appear to be reproduced in this murine obesity-associated model.

Griffin and colleagues fed C57Bl6/6j mice a low- or high-fat diet until 54 weeks of age. Remarkably, although an inbred strain was used, a dichotomy within the

\footnotetext{
*Correspondence: P.vanderkraan@reuma.umcn.nl

Experimental Rheumatology and Advanced Therapeutics, Radboud University Nijmegen Medical Centre, 6525 GA Nijmegen, The Netherlands

Current address: Nijmegen Centre for Molecular Life Sciences (NCMLS), Geert Grooteplein 28, 6525 GA Nijmegen, The Netherlands
}

high-fat fed mice was observed. Mice on the high-fat diet could be divided into 'low gainer' (LG; low body mass and fat) and 'high gainer' (HG; high body mass and fat) populations depending on their extent of weight gain. An explanation for this enigmatic finding in an inbred mouse strain is not obvious, but differences in social status and related elevated stress levels might play a role [2]. The HG mice showed an increased incidence of knee OA, mainly indicated by loss of cartilage proteoglycans. So high susceptibility to obesity was associated with OA-like changes in the knee joint. Since the HG phenotype seems to be the consequence of a combination of both a highfat diet and high stress levels, one might speculate that high stress levels play a role in OA development in these animals. It can be anticipated that high stress levels will have an effect on corticosteroid levels and activity of the mice.

Griffin and colleagues further tested whether diet, fat deposition and OA were associated with locomotor activity and grip strength. A high-fat diet was negatively correlated with grip strength, even when percent body fat and OA were taken into account. Motor coordination on a turning rod was decreased, in proportion to percent body fat, in mice with the high-fat diet. Whereas spontaneous locomotion was not changed, peak vertical force on a force plate was negatively related to OA severity of the knee. These findings appear to correspond to observations in human OA patients. Electromyographic patterns of the knee suggest changes in motor unit function associated with early OA and the magnitude of this change was higher in more severe cases [3]. So early changes in locomotor function are associated with $\mathrm{OA}$ development in both humans and mice.

Interestingly, the high-fat diet was associated with hyperalgesia, corresponding to symptomatic findings in human OA, but unrelated to structural OA development in the mice studied. An absence of consistent correlations between pain and radiographic and magnetic resonance imaging findings consistent with $\mathrm{OA}$ have also been found in human knee OA [4]. A relationship between overall sensitization to pain and knee OA was recently reported [5]. However, a high-fat, low-carbohydrate diet 
(ketogenic diet) was shown to reduce thermal pain [6]. Apparently diet and $\mathrm{OA}$ influence the sensitivity to painful stimuli not only in humans but also in this murine model, suggesting that similar mechanisms are acting in both species.

As well as hyperalgesia, anxiety-like behavior was associated with a high-fat diet and percentage body fat in mice. It is intriguing that it is becoming increasingly clear that a high body mass index and body fat are associated with depression and anxiety in humans [7]. As can be concluded from the study of Griffin and colleagues, this is not only the case in human subjects but also in small rodents. Since in humans it is suggested that psychological factors, such as self-image, influence eating behavior, the observations in mice point to a more physiological cause of the altered behavior, hypothetically related to stress level differences between individuals.

The findings of Griffin and colleagues are fascinating since they show that many of the extra-articular alterations that are found in human OA can be reproduced in a small animal model of OA. Changes in locomotor function, sensitization to pain and depression/anxietylike symptoms were found in their high-fat-induced model of OA. These observations indicate that a small animal model can represent more aspects of OA pathophysiology than simply the structural changes in the affected joint. Moreover, their study clearly demonstrates that $\mathrm{OA}$ is not only a cartilage disease, not even a disease of the whole joint, but that the entire organism appears to be involved in the disease process itself and the expression of its related symptoms, both in mice and humans.
Abbreviations

$H G=$ high gainer; $\mathrm{OA}=$ osteoarthritis

\section{Competing interests}

The author declares that they have no competing interests.

Published: 28 July 2010

\section{References}

1. Griffin TM, Fermor B, Huebner JL, Kraus VB, Rodriguiz RM, Wetsel WC, Cao L, Setton LA, Guilak F: Diet-induced obesity differentially regulates behavioral, biomechanical, and molecular risk factors for osteoarthritis in mice. Arthritis Res Ther 2010, 12:R130.

2. Kuo LE, Czarnecka M, Kitlinska JB, Tilan JU, Kvetnansky R, Zukowska Z: Chronic stress, combined with a high-fat/high-sugar diet, shifts sympathetic signaling toward neuropeptide $Y$ and leads to obesity and the metabolic syndrome. Ann N Y Acad Sci 2008, 1148:232-237.

3. Ling SM, Conwit RA, Talbot L, Shermack M, Wood JE, Dredge EM, Weeks MJ, Abernethy DR, Metter EJ: Electromyographic patterns suggest changes in motor unit physiology associated with early osteoarthritis of the knee. Osteoarthritis Cartilage 2007, 15:1134-1140.

4. Barker K, Lamb SE, Toye F, Jackson S, Barrington S: Association between radiographic joint space narrowing, function, pain and muscle power in severe osteoarthritis of the knee. Clin Rehabil 2004, 18:793-800.

5. Arendt-Nielsen L, Nie H, Laursen MB, Laursen BS, Madeleine P, Simonsen OH, Graven-Nielsen T: Sensitization in patients with painful knee osteoarthritis. Pain 2010, 149:573-581

6. Ruskin DN, Kawamura M, Masino SA: Reduced pain and inflammation in juvenile and adult rats fed a ketogenic diet. PLoS One 2009, 4:e8349.

7. Hillman JB, Dorn LD, Huang B: Association of anxiety and depressive symptoms and adiposity among adolescent females using dual energy X-ray absorptiometry. Clin Pediatr (Phila) 2010, 49:671-677.

doi:10.1186/ar3082

Cite this article as: ven der Kraan PM: Osteoarthritis and a high-fat diet: the full 'OA syndrome' in a small animal model. Arthritis Research \& Therapy 2010, $12: 130$. 